\title{
JUXTACORTICAL CHONDROSARCOMA
}

\author{
Fritz Schajowicz, Buenos Aires, Argentina \\ From the Latin-American Registry of Bone Pathology, Buenos Aires
}

\begin{abstract}
The features are described of seven cases of "juxtacortical" chondrosarcoma, the term introduced by Jaffe for a rare but distinct entity and now accepted in the World Health Organisation classification as preferable to the term "periosteal" chondrosarcoma. In all cases the lesion involved the shaft of a long bone, most often the femur, and in two cases two different long bones were affected. Six of the seven patients were male and all were in the second decade. The characteristic appearance was that of a small tumour adjacent to the cortex with areas of spotty calcification often accompanied by radiating bone spicules perpendicular to the bone shaft and a typical Codman's triangle. Histologically all the tumours showed a cartilaginous lobular pattern, well limited on the surface and seldom infiltrating the cortex; areas of spotty calcification and enchondral ossification were often present but tumour osteoid and bone were conspicuously absent. Despite the ominous histological aspect, the prognosis proved to be relatively favourable compared with the usual central chondrosarcoma of a similar grade of malignancy.
\end{abstract}

In 1955 Lichtenstein reported as a rare entity, for which he used the term "periosteal" chondrosarcoma, a rather small lesion of the upper shaft of the humerus in a young adult which in part had a shell of periosteal new bone; no recurrence had occurred one year after block excision. Another lesion, this one of the femoral shaft, was first regarded as a periosteal chondrosarcoma but showed in subsequent specimens other features "clearly stamping it as a cartilage-containing osteogenic sarcoma". In 1958 Jaffe stated that "occasionally one observes a lesion which represents the malignant counterpart of the juxtacortical chondroma". He thus introduced the more flexible term "juxtacortical" chondrosarcoma, emphasising its relatively good prognosis even after local resection. In 1965 Cooper reported a case of "juxtacortical" chondrosarcoma of the shaft of the ulna in a youth of seventeen, with a recurrence in the local subcutaneous tissue four months after a local resection but no further recurrence after seventeen months. In 1967 Schajowicz and Bessone reported two cases of "juxtacortical" chondrosarcoma, one with similar metachronous lesions in the shafts of a fibula and a femur; these are included here as Cases 4 and 5.

Juxtacortical chondrosarcoma has been defined in the World Health Organisation classification as a "malignant cartilage-forming tumour arising from the external surface of bone, usually characterised by welldifferentiated cartilage with extensive areas of enchondral ossification" (Schajowicz, Ackerman and
Sissons 1972). It was also stated that this rare lesion possibly represents a cartilage counterpart of juxtacortical osteosarcoma. The better prognosis than that for the usual central type of chondrosarcoma was emphasised, and the distinction was made from a chondrosarcoma arising from the cartilage cap of an osteochondroma, which is classified by most authors as "peripheral" chondrosarcoma (Henderson and Dahlin 1963; Lichtenstein 1965; Spjut, Dorfman, Fechner and Ackerman 1971). In our opinion most of the cases from the Mayo Clinic reported recently by Unni, Dahlin and Beabout (1976) as a distinct entity called "periosteal osteogenic sarcoma" were in reality juxtacortical chondrosarcomata. Histologically all such cases were chondroblastic osteogenic sarcomata and had clinical and pathological features very similar to our own.

Five more cases, all in young adults under twentyand among them another case with two metachronous lesions in different long bones-have now been studied in our laboratory, and we hold the complete resection or amputation specimens, one dating back to 1946 . With the exception of one patient, all have been followed for long periods of time and are in good health from thirty down to three years after the initial surgical treatment. On the basis of this experience, gleaned from over 2,000 cases of primary malignant tumour filed at the Centre for Osteoarticular Pathology, we are now reporting in some detail the features of this rare tumour in order to stress the appropriate management. 


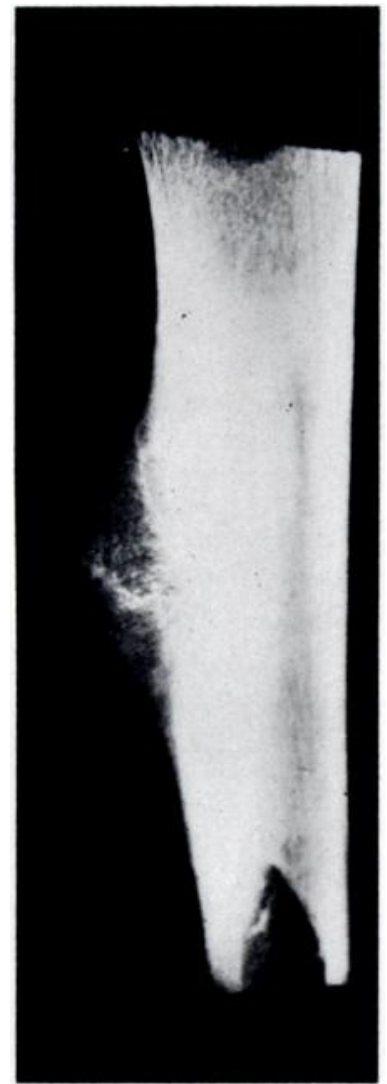

Fig. 1

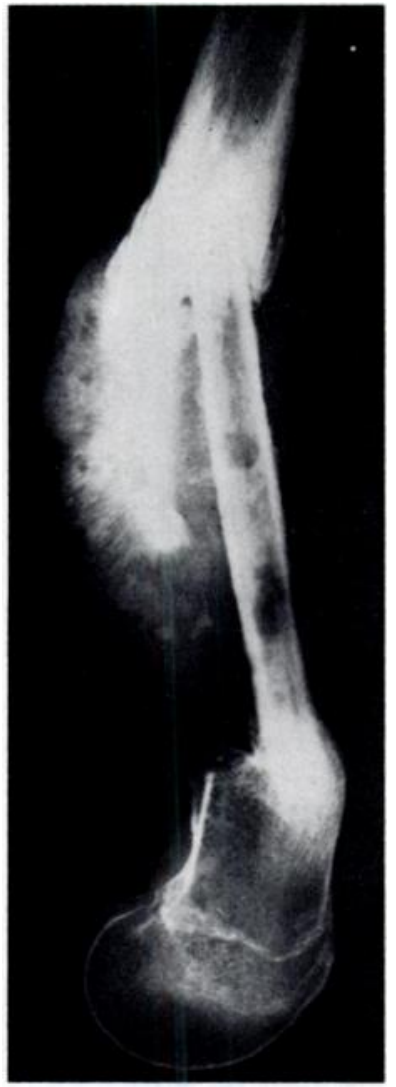

Fig. 2

Case 1. Femur.

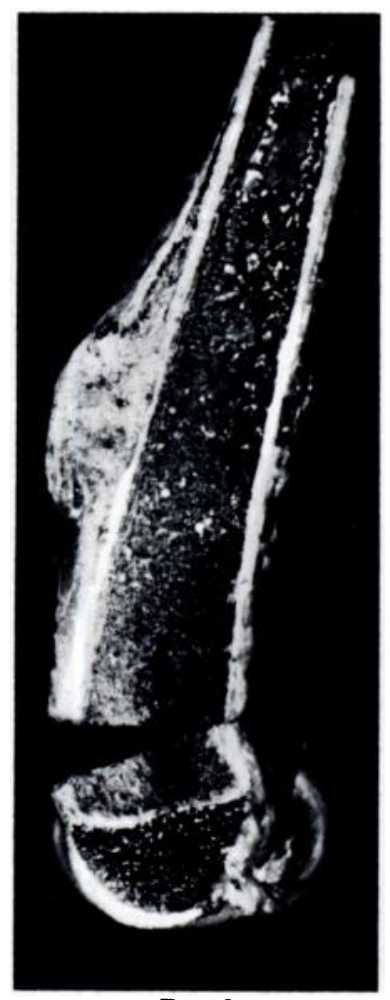

Fig. 3

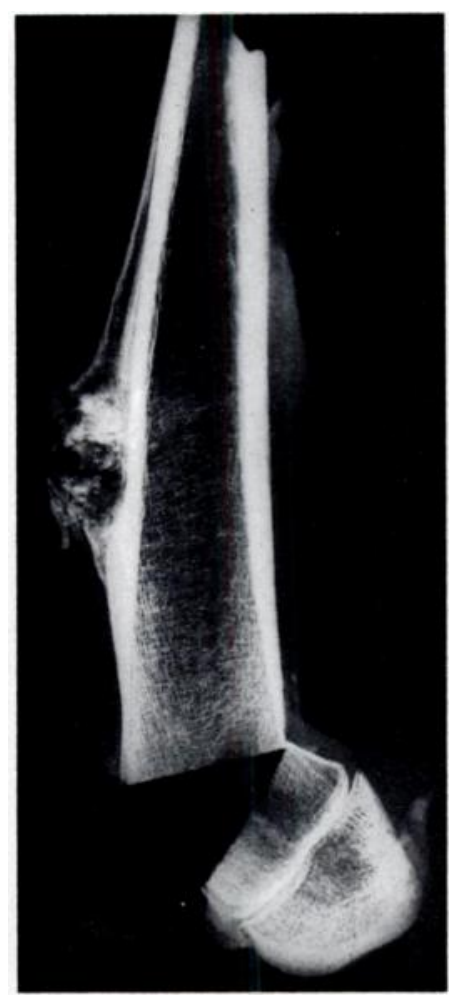

Fig. 4

Case 2. Femur.

\section{CASE REPORTS}

Case 1-A boy of fifteen was admitted in October 1946 complaining of pain and swelling over the middle of the right thigh for three months. In November 1946, after a needle biopsy, block resection of a small soft-tissue tumour together with a segment of the related femoral shaft was performed, followed by an autogenous fibular graft. Radiographs of the specimen showed spotty calcification and radiating bone spicules perpendicular to the cortex (Fig. 1). Histologically a diagnosis of chondrosarcoma of medium-grade malignancy was made.

By April 1947 a local recurrence had widely invaded the soft tissues, the bone graft and the lower end of the upper fragment of femur (Fig. 2). Subcapital amputation was performed. Histologically the malignancy was now highgrade. Nevertheless, at review in December 1976 this patient was in excellent general health, thirty years after the first operation.
Case 2-A boy of sixteen had pain and swelling of the left thigh for several months before admission. An open biopsy had already been performed in another hospital in July 1954 . Radiographs showed a small juxtacortical tumour with radiating bone spicules but without obvious involvement of the cortex. A small defect from the biopsy and typical Codman's triangles were observed. Total resection of the lowermost third of femur was carried out (Figs. 3 and 4), followed by a massive homogenous replacement. Histologically the tumour was a chondrosarcoma of medium-grade malignancy.

By August 1965 a pseudarthrosis of the upper end of the replacement had developed, for which an autogenous bone graft was applied after fixation by a Kuntscher nail. In October 1972, eighteen years after the first operation and seven years after the second, the grafts were soundly consolidated with no sign of recurrence.

Case 3-A young woman of nineteen was admitted in August 1954 for pain and swelling over the midshaft of the left tibia, present for about two months. Radiographs showed a zone of irregular and spotty calcification in relation to the shaft, which was slightly enlarged. In August 1954 a wide excision of the tumour and related cortex was carried out. The gross specimen showed penetration by the tumour into the outer part of the spongiosa. Histologically the lesion was a chondrosarcoma of moderate malignancy. Unfortunately the further history of this patient is not known. 


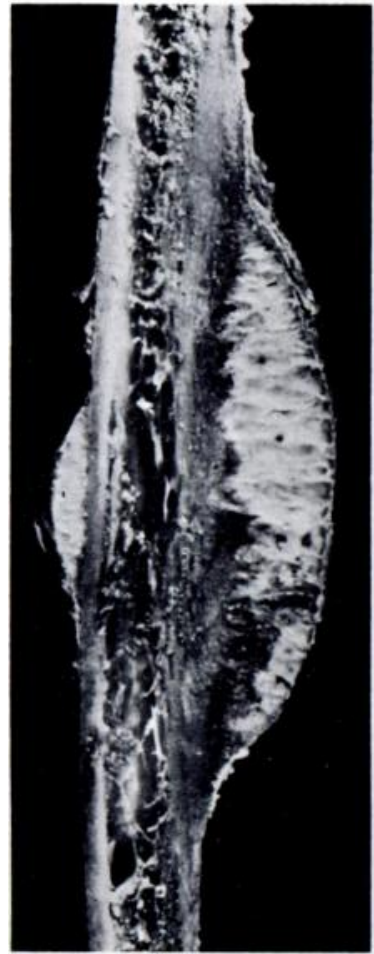

Fig. 5

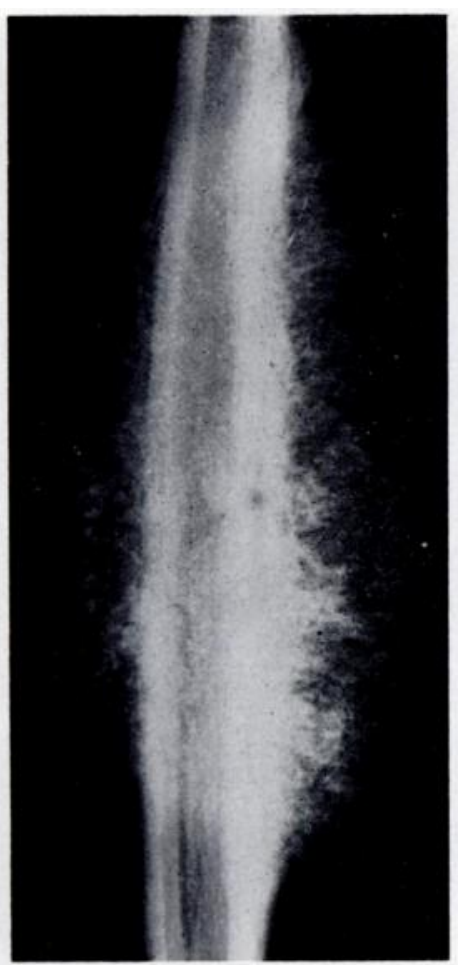

FiG. 6

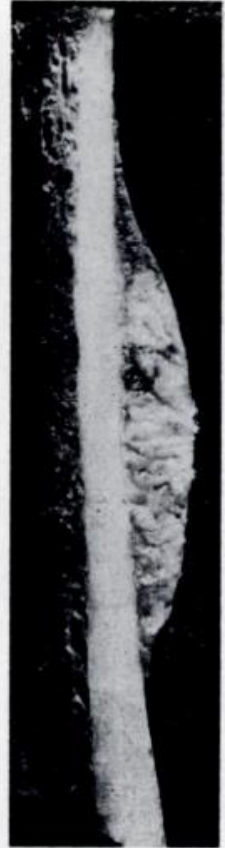

Fig. 7

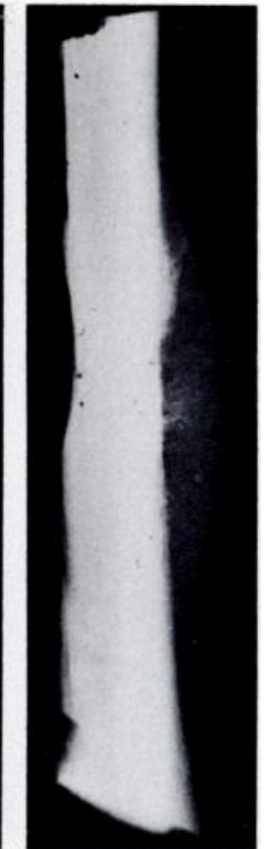

Fig. 8

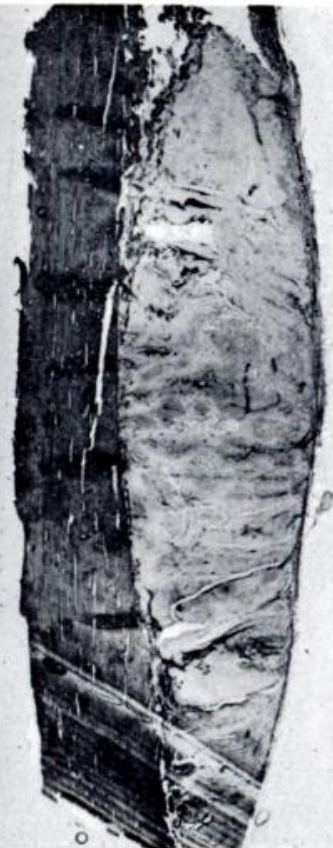

Fig. 9
Case 4. Femur.

Case 4. Fibula.

Case 4-A boy of sixteen noted a hard painful swelling in the middle third of the left leg after a minor contusion two weeks before attending in February 1964. The mass was diffuse and firm, and apparently arose from the fibula. The radiographs showed an extraosseous tumour with spotty calcification and bone spicules giving a sun-ray appearance. The tumour was apparently of periosteal or parosteal origin and did not seem to have invaded the medulla. An aspiration biopsy was done immediately and a diagnosis was made of chondrosarcoma of medium-grade malignancy. A block resection of 21 centimetres of the fibular shaft, including the neighbouring muscle, was performed (Figs. 5 and 6). The specimen showed a lobulated tumour with a sharply defined surface. On longitudinal section the juxtacortical location was quite evident; the tumour had partly destroyed the enlarged cortex on one side only, without invading the medulla.

Histologically, this was a chondrosarcoma with some rather cellular areas; it was not well differentiated and was of moderate malignancy. Extensive zones of calcification were present, often accompanied by enchondral ossification, but undoubted tumour bone formation was not seen.

The patient recovered well from his fibular lesion. However, in August 1966 he returned with a firm painful mass over the postero-lateral aspect of the middle third of his thigh, again on the left side, with no history of trauma. Radiographs showed only slight irregularity of the posterior cortex.

Aspiration biopsy was performed at once. Two weeks later the tumour and underlying cortex were resected en bloc and the defect was repaired with a bone graft. The specimen again revealed a typical juxtacortical chondrosarcoma (Figs. 7 to 9). Histologically this tumour was more malignant (grade 3) than the fibular lesion, with more atypical cells.

Three months later, while walking in his plaster cast, the patient fractured the femur just above the bone graft, which was reinforced with tibial bone. Three years later, in October 1969, a large recurrence of the femoral tumour appeared (Figs. 10 and 11) and aspiration biopsy showed high malignancy. Disarticulation through the hip was then performed. This patient was last seen in December 1976 when he was in excellent health over twelve years from the onset of symptoms and seven years after the disarticulation.

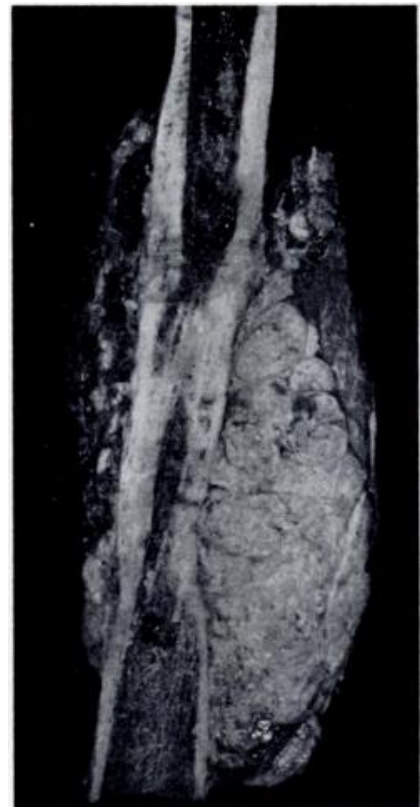

Fig. 10

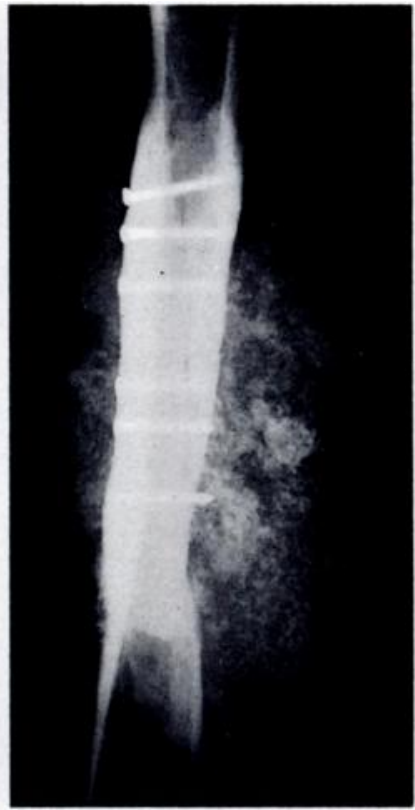

Fig. 11
Case 4. Femur, recurrence. 


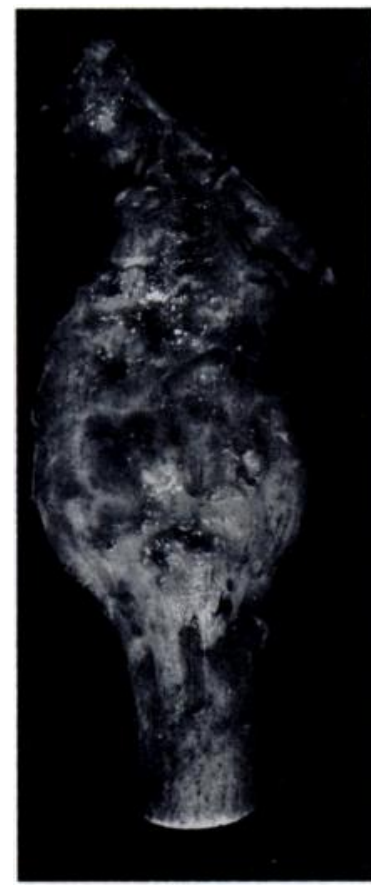

FIG. 12

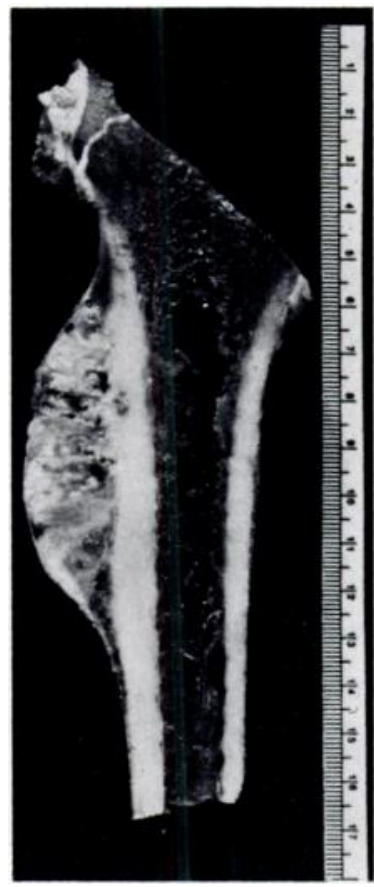

Fig. 13

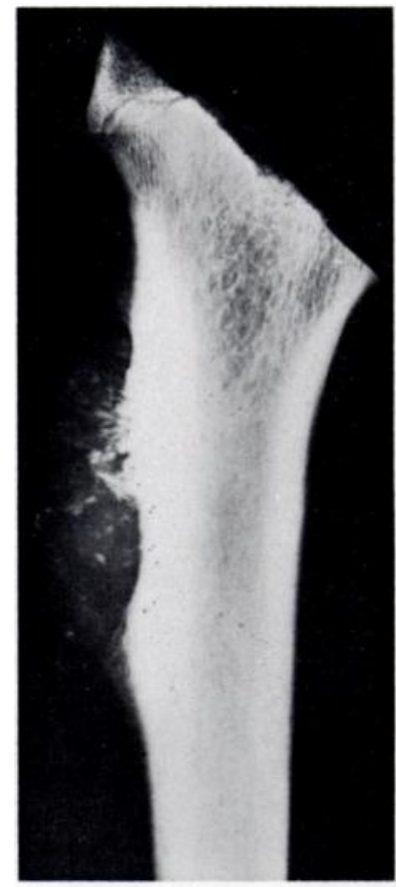

Fig. 14

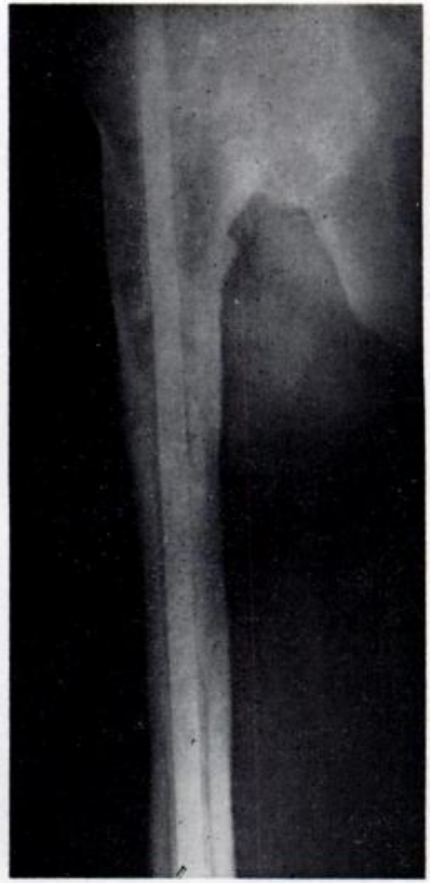

Fig. 15

Case 5. Femur.

Case 5-A boy of seventeen, twin brother of the patient in Case 4, was admitted nine months after him, in November 1964, for a firm fixed mass in the uppermost third of the right thigh which had appeared some months after a local contusion. Radiographs showed a lesion of the upper shaft, with irregular areas of spotty calcification, an appearance like Codman's triangles and slight thickening of the cortex. Aspiration biopsy led to a diagnosis of chondrosarcoma of medium-grade malignancy. A wide block resection, 18 centimetres long, was performed a week later by Professor Carlos Ottolenghi, the defect being repaired by a homogenous bone-bank graft fixed with a Kuntscher nail.

The resected specimen was surrounded by a lobulated, sharply-defined tumour of hard consistency (Fig. 12). Longitudinal section showed the tumour to be clearly extraosseous and juxtacortical, apparently of subperiosteal and not parosteal origin (Fig. 13). It was greyish-blue and looked like cartilage. It had slightly invaded the underlying cortex but not the medulla. Radiographs again showed the characteristic spotty calcification (Fig. 14). Further sections confirmed the earlier diagnosis. The periphery of the lobules contained undifferentiated round cells with relatively little matrix; toward the centre cartilaginous differentiation was evident, with extensive areas of calcification and enchondral bone formation. The diagnosis was juxtacortical (periosteal) chondrosarcoma of grade 2 to 3.

The graft consolidated perfectly (Fig. 15) and the patient was in excellent general health in December 1976, twelve years after the initial surgical treatment.

Case 6-A boy of eleven was admitted in July 1964 with a painless swelling over the uppermost third of the right ulna for three months. The tumour was hard and fixed to bone. The radiographs showed a dense, calcified, well-defined juxtacortical mass, and aspiration biopsy yielded a diagnosis of chondrosarcoma.
In August 1964, a wide block resection 8 centimetres long was performed (Figs. 16 to 18) followed by an autogenous bone graft. In September 1967 a pseudarthrosis appeared and successful autogenous grafting was carried out. However, in March 1969 a second tumour appeared, this one over the uppermost third of the right fibula shortly after a local contusion. Radiographs showed a large juxtacortical mass with radiating bone spicules and a typical Codman's triangle at the lower end. In June 1969 open biopsy was followed by wide block resection (Figs. 19 to 21). The patient was well and without recurrence in July 1970, six years after the first and over one year after the second resection.

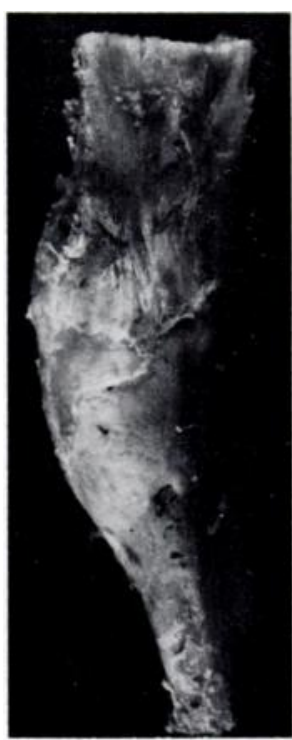

Fig. 16

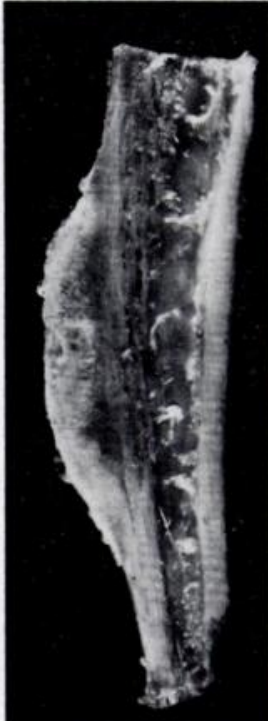

FIG. 17

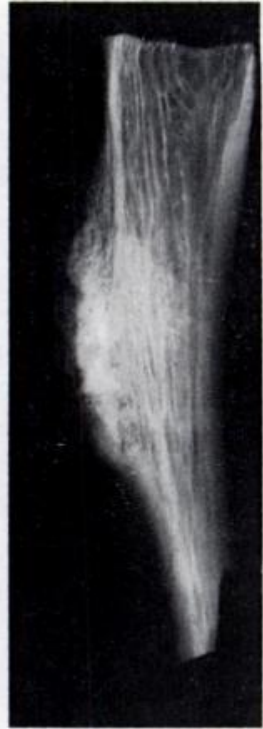

Fig. 18
Case 6. Ulna. 

complaining of a painful hard swelling over the middle of the left thigh for two months. Radiographs showed a small softtissue mass with spotty calcification and radiating bone spicules producing a slight erosion of the subjacent cortex and typical Codman's triangles. A wide block resection of $\mathbf{1 8}$ by Professor Ottolenghi, and repaired by a homogenous bone graft fixed with a Kuntscher nail. The gross specimen showed the characteristic chondroid aspect of the tumour with underlying sclerotic cortex (Fig. 22). A radiograph of the specimen showed the typical aspect of juxtacortical chondrosarcoma (Fig. 23).

In August 1976 the patient fractured the graft while walking; intramedullary nailing was again performed. The graft soon consolidated and the patient was well and with no sign of recurrence in December 1976, three and a half years after the block resection (Fig. 24).
Case 7-A boy of seventeen was admitted in March 1973 centimetres of the femoral shaft was immediately performed zones of calcification and enchondral ossification near the

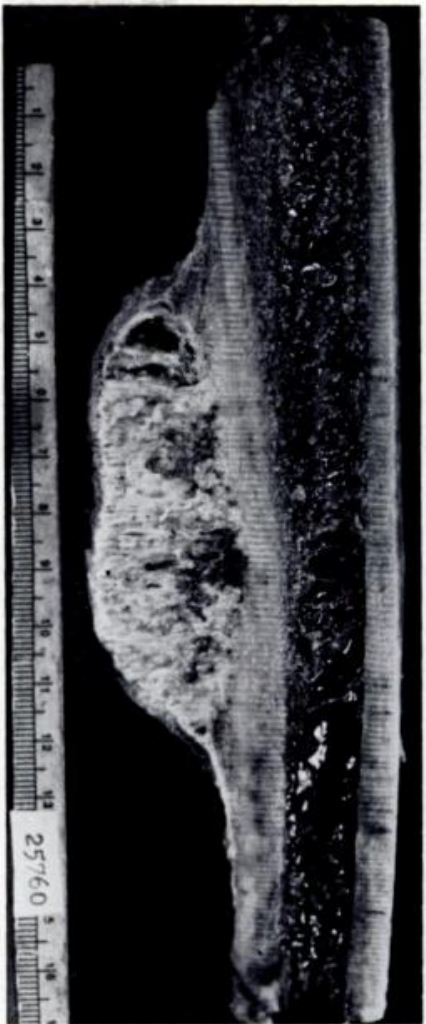

Fig. 22

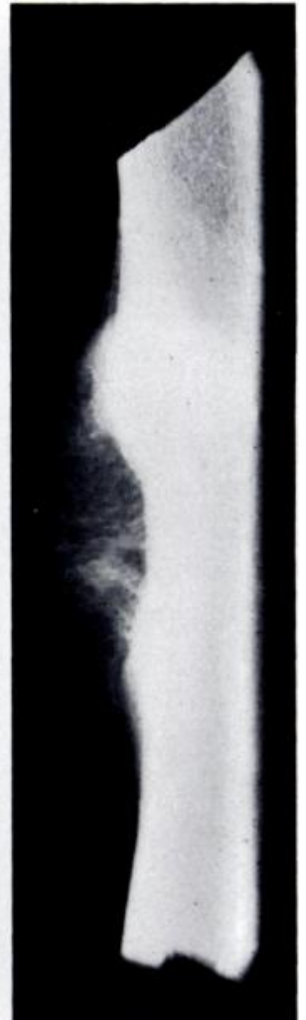

FIG. 23
Case 6-continued

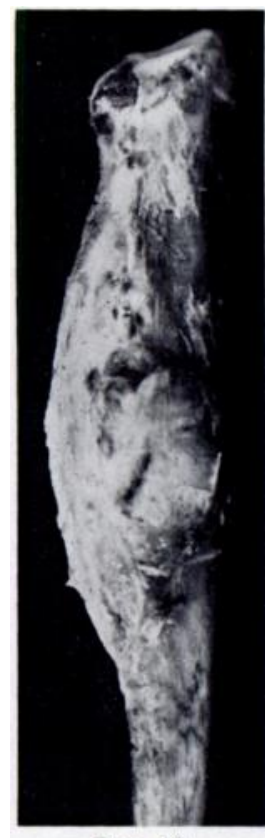

FIG. 19

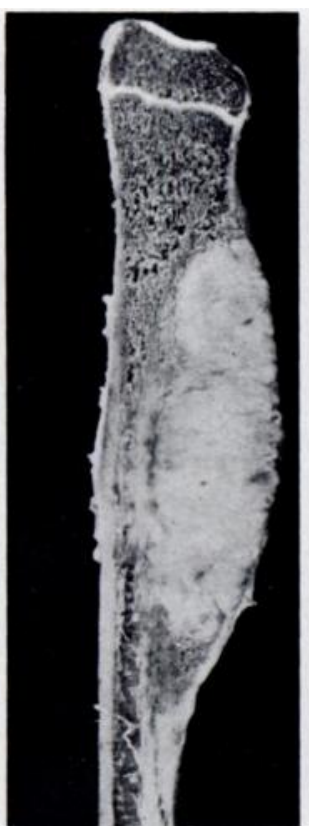

FIG. 20

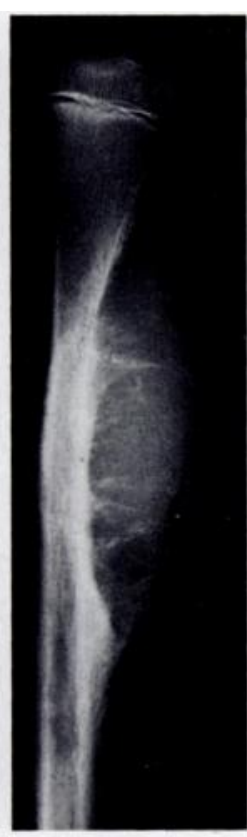

FIG. 21
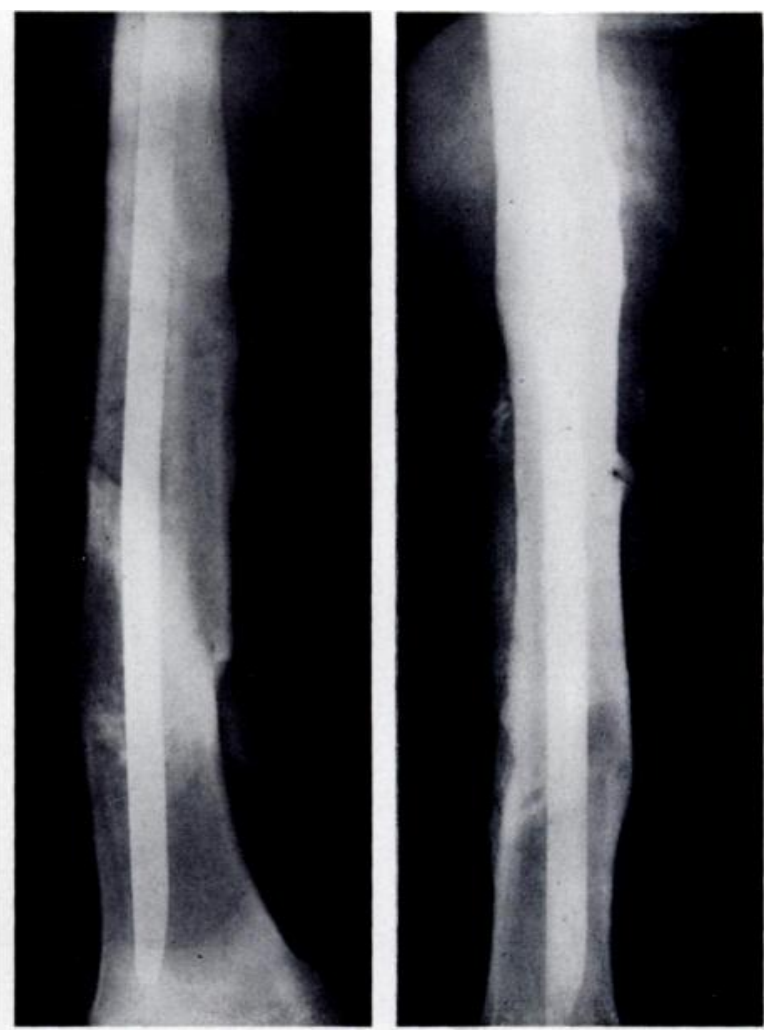

FIG. 24

Figures 22 to $24-$ Case 7. Femur. 


\section{CLINICAL CHARACTERISTICS}

The seven patients were aged between eleven and nineteen years, and six were male. All had noted a swelling over the shaft of a long bone which had appeared a few weeks or months after a slight injury in three cases and was painful in five of the seven. Two patients developed two lesions in different long bones on the same side of the body (fibula and femur in Case 4; ulna and fibula in Case 6), the second tumour appearing two and three years respectively after surgical treatment of the first. Including these second tumours, the femur was involved in five cases, the fibula in two, the ulna in one and the tibia in one.

Radiographic features-The appearances were almost uniformly those of a juxtacortical soft-tissue mass of moderate size with more or less conspicuous and irregular areas of spotty calcification, usually accompanied by very characteristic radiating bone spicules which generally did not reach the surface of the tumour mass and gave a sun-ray appearance. The lesions were always on the shaft of a long tubular bone and in most cases there was a typical Codman's triangle. The subjacent cortex was sometimes eroded, concave and slightly altered or thickened.

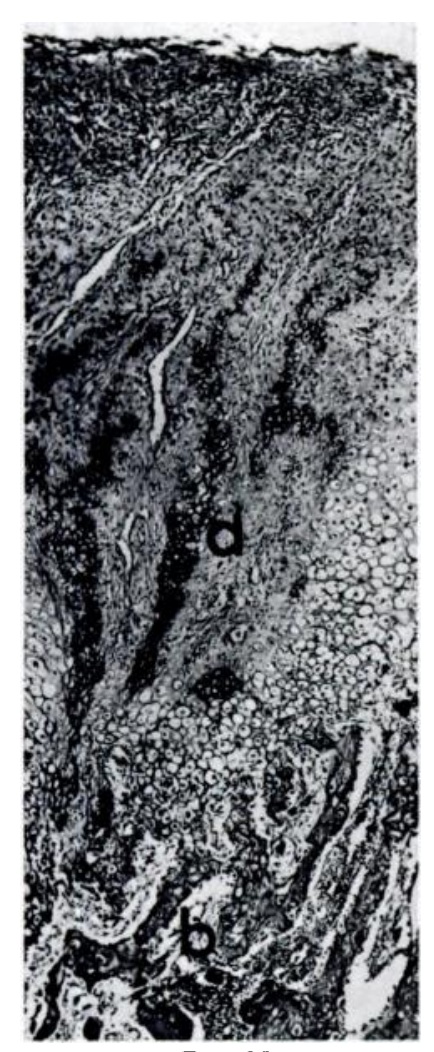

Fig. 25

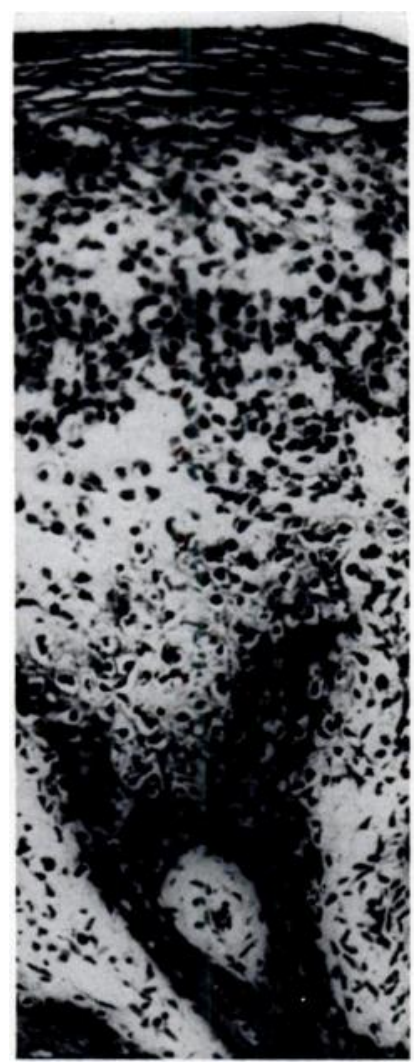

Fig. 26
Figure 25-The characteristic zones of cartilage calcification (a) and enchondral ossification $(b) . \quad(\times 40$.) Figure $26-A$ cellular area of undifferentiated cells and perpendicular trabeculae of calcified cartilage. $(\times 100$.
Pathological features-The macroscopic appearances were very similar in all cases. The resected specimens showed a slightly lobulated tumour with a well-defined surface, generally covered by a thick fibrous layer which appeared to be the continuation of the periosteum elevated at the margins of the tumour. The lesion was generally not more than 7 to 8 centimetres long. At the surface the consistency was soft, the deeper zones being firm or hard. On longitudinal section the extraosseous, juxtacortical and subperiosteal location of the tumour was quite evident. As for colour, the tumour was generally the greyish-blue so typical of cartilaginous tissue, with more or less abundant yellow zones of spotty calcification alternating with reddish haemorrhagic zones close to the cortex and corresponding with enchondral ossification.

The underlying cortex was to some extent eroded causing a concavity in a few cases, and was more or less thickened beyond the tumour mass. The medullary cavity was not involved except in one case (Case 3). At each end of the tumour the periosteum was elevated, covering the surface of the lesion, and in most cases a Codman's triangle was observed. This demonstrated the subperiosteal location of the tumour and indicated a probable periosteal origin.

Histologically, all the tumours showed very similar features, with a conspicuous lobular arrangement perpendicular to the bone surface (Fig. 25). The periphery of the lobules contained more undifferentiated cells of roundish or spindle shape with relatively little intercellular matrix, and was covered by a fibrous layer continuous with the periosteum (Fig. 26). Towards the centre of the lobules cartilaginous differentiation was evident, with more abundant hyaline matrix. In most cases a more or less conspicuous cellular atypism was observed in these areas, with the presence of one or more plump nuclei, sometimes monstrous or bizarre, indicating a moderate to high-grade malignancy (Fig. 27). This was even more conspicuous in the two cases of local recurrence. In only two cases was the grade of malignancy low to moderate at the first histological study.

In all tumours more or less extensive zones of calcification were present (Figs. 25 and 26), generally accompanied by enchondral ossification in the juxtacortical areas and starting perpendicularly from the underlying cortex (Figs. 25 and 28). However, clear formation of tumour osteoid or bone was not seen, which excludes the diagnosis of so-called "periosteal osteogenic sarcoma" (Unni et al. 1976).

\section{DISCUSSION}

We prefer the use of the term "juxtacortical chondrosarcoma" introduced by Jaffe and accepted as a distinct malignant cartilage tumour in the W.H.O. classification. Although its development in close relation to and 
beneath the periosteum makes a periosteal origin highly probable in most cases, the term "juxtacortical" seems to us more appropriate than "periosteal" (Lichtenstein and Hall 1952) because it is not only less compromising with respect to the site of origin, but also because it is more descriptive, emphasising better the close relationship of the tumour tissue to the adjacent bone cortex.

\section{Differential diagnosis}

Juxtacortical (periosteal) chondroma is more common than juxtacortical chondrosarcoma; it involves the long bones of the hand and foot more frequently, but also the long limb bones. Although at times it may show some nuclear atypism, the cartilage cells are nearly always unicellular and the nuclei are rarely plump. The few cases of recurrences (Nosanchuk and Kaufer 1969) possibly stem from their incomplete excision or from failure to recognise malignancy.

Peripheral chondrosarcoma is a not infrequent malignant change arising in the cartilage cap of an osteochondroma. It is generally found in the metaphysial region of a long bone or in a flat bone, especially the ilium; it often occurs after the second decade; and it has a pedicle of cancellous bone continuous with the spongiosa of the underlying medullary cavity.

The main problem is the so-called "periosteal osteogenic sarcoma" described recently by Unni et al.

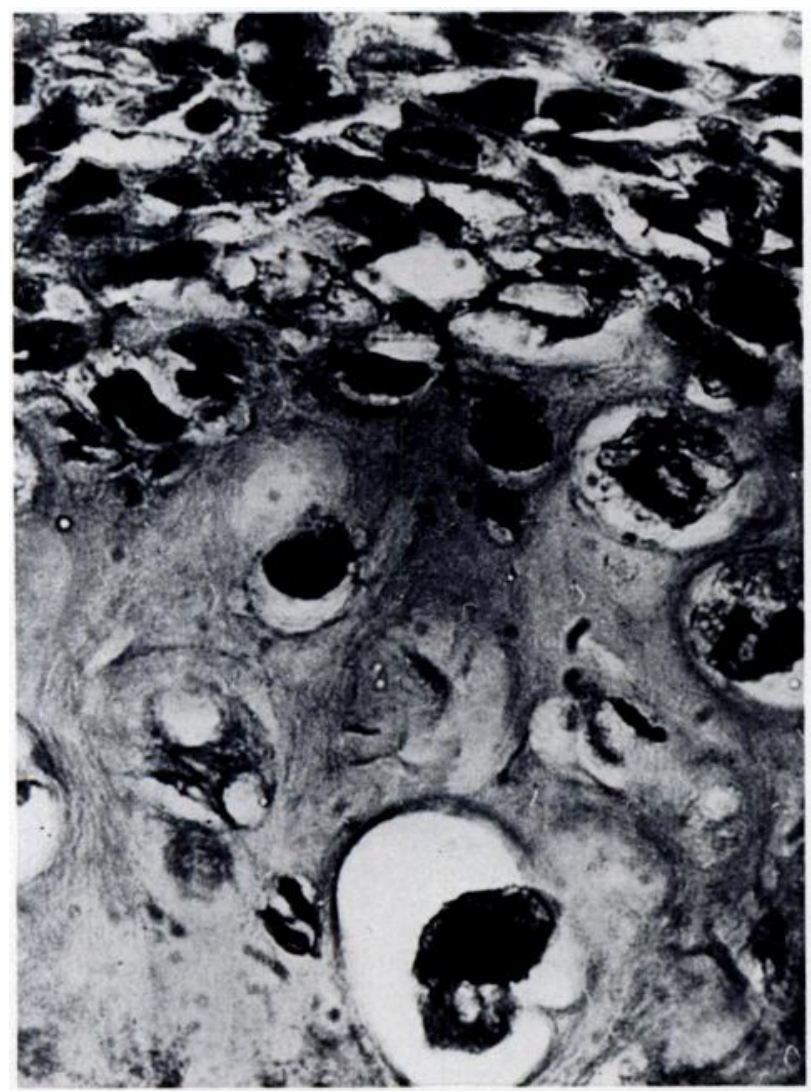

Fig. 27
(1976) as a new and distinct entity with particular differences from "parosteal osteogenic sarcoma". Histologically, all these cases were regarded as malignant chondroblastic osteogenic sarcoma of relatively high grade (grade 2 to 3 ). The radiographic and pathological features were so similar to those of our cases that it seems to us that at least a great part of theirs were really examples of juxtacortical (periosteal) chondrosarcoma. We have never seen in our cases clearly evident production of malignant osteoid, forming "fine lace-like osteoid" with or without mineralisation. The only illustration in their article, supposed to demonstrate this pattern, shows in our opinion some scanty cartilage matrix and not tumour osteoid. With this comment we do not intend to deny the existence of quite exceptional cases of a "periosteal" osteogenic sarcoma or osteosarcoma of chondroblastic type and juxtacortical location, with clinical and pathological features different from the conventional "juxtacortical osteosarcoma". Indeed, we have observed recently one case of this type.

In short, juxtacortical chondrosarcoma would seem to represent the malignant counterpart of juxtacortical chondroma and may be considered, in accordance with Jaffe, as the cartilage analogue of juxtacortical (parosteal) osteosarcoma. The prognosis seems to be much better than with the usual central chondrosarcoma of a similar histological grade of malignancy.

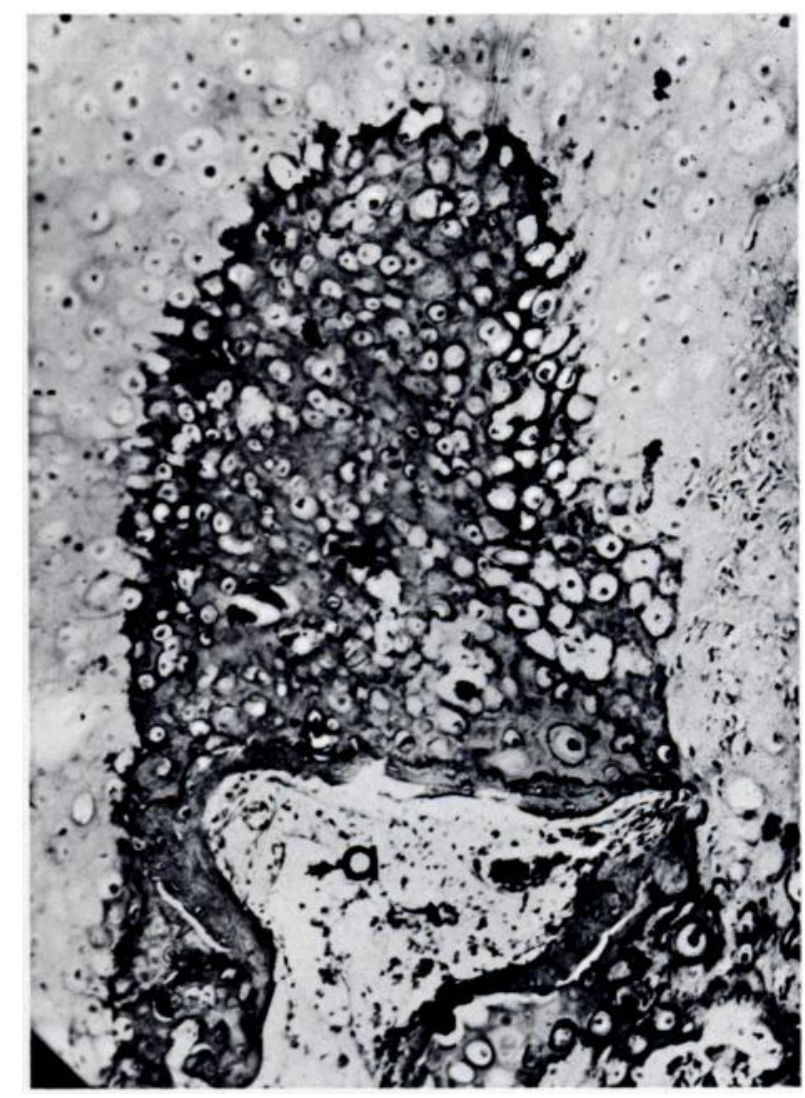

Fig. 28

Figure 27-Cartilage cells with monstrous bizarre nuclei. $(\times 480$.) Figure 28 -Cartilage tumour tissue with an advancing zone of enchondral ossification $(\mathrm{a})$. $(\times 100$. 


\section{ACKNOWLEDGEMENT}

The author wishes to express thanks to Dr I. Ayerza, Dr J. E. Bessone, Professor C. E. Ottolenghi, Dr J. Piqué and Dr S. Schächter, for permission to publish their cases.

\section{REFERENCES}

Cooper, R. R. (1965) Juxtacortical chondrosarcoma. Journal of Bone and Joint Surgery, 47-A, 524-528.

Henderson, E. D., and Dahlin, D. C. (1963) Chondrosarcoma of bone. A study of two hundred and eighty-eight cases. Journal of Bone and Joint Surgery, 45-A, 1450-1458.

Jaffe, H. L. (1958) Tumors and Tumorous Conditions of the Bones and Joints. London: Henry Kimpton.

Lichtenstein, L. (1955) Tumors of periosteal origin. Cancer, 8, 1060-1069.

Lichtenstein, L. (1965) Bone tumors. Third edition. St Louis: C. V. Mosby Company.

Lichtenstein, L., and Hall, J. E. (1952) Periosteal chondroma. Journal of Bone and Joint Surgery, 34-A, 691-697.

Nosanchuk, J. S., and Kaufer, H. (1969) Recurrent periosteal chondroma. Journal of Bone and Joint Surgery, 51-A, 375-380.

Schajowicz, F., and Bessone, J. E. (1967) Chondrosarcoma in three brothers. Journal of Bone and Joint Surgery, 49-A, 129-141.

Schajowicz, F., Ackerman, L. W., and Sissons, H. A. (1972) Histological Typing of Bone Tumours. International Histological Classification of Tumours, No. 6. Geneva: W.H.O.

Spjut, H. J., Dorfman, H. D., Fechner, R. E., and Ackerman, L. V. (1971) Tumors of Bone and Cartilage. Atlas of Tumor Pathology. Second Series, Fascicle 5. Washington, D.C.: Armed Forces Institute of Pathology.

Unni, K. K., Dahlin, D. C., and Beabout, J. W. (1976) Periosteal osteogenic sarcoma. Cancer, 37, 2476-2485. 